\title{
The radio to $\mathrm{TeV}$ orbital variability of the microquasar LS I +61303
}

\author{
V. Bosch-Ramon ${ }^{1,2}$, J. M. Paredes ${ }^{1}$, G. E. Romero ${ }^{3,4, \star}$, and M. Ribó ${ }^{1}$ \\ 1 Departament d'Astronomia i Meteorologia, Universitat de Barcelona, Martí i Franqués 1, 08028 Barcelona, Catalonia, Spain \\ e-mail: jmparedes@ub.edu; mribo@am.ub.es \\ 2 Max Planck Institut für Kernphysik, Heidelberg 69117, Germany \\ e-mail: vbosch@mpi-hd.mpg.de \\ 3 Instituto Argentino de Radioastronomía, CC 5, (1894) Villa Elisa, Buenos Aires, Argentina \\ e-mail: romero@iar.unlp.edu.ar \\ 4 Facultad de Ciencias Astronómicas y Geofísicas, UNLP, Paseo del Bosque, 1900 La Plata, Argentina \\ e-mail: romero@fcaglp.unlp.edu.ar
}

Received 14 June 2006 / Accepted 19 September 2006

ABSTRACT

\begin{abstract}
Context. The microquasar LS I +61 303 has recently been detected at TeV energies by the Cherenkov telescope MAGIC, presenting variability on timescales similar to its orbital period. This system has been intensively observed at different wavelengths during the last three decades, showing a very complex behavior along the orbit.

Aims. We aim to explain, using a leptonic model in the accretion scenario, the observed orbital variability and spectrum from radio to $\mathrm{TeV}$ energies of LS I +61303.

Methods. We apply a leptonic model based on accretion of matter from the slow inhomogeneous equatorial wind of the primary star, assuming particle injection proportional to the accretion rate. The relativistic electron energy distribution within the binary system is computed taking into account convective/adiabatic and radiative losses. The spectral energy distribution (SED) has been calculated accounting for synchrotron and (Thomson/Klein Nishina -KN-) inverse Compton (IC) processes and the photon-photon absorption in the ambient photon fields. The angle dependence of the photon-photon and IC cross sections has been considered in the calculations. Results. We reproduce the main features of the observed light curves from LS I +61 303 at radio, X-rays, high-energy (HE), and very high-energy (VHE) gamma-rays, and the whole spectral energy distribution.

Conclusions. Our model is able to explain the radio to $\mathrm{TeV}$ orbital variability taking into account that radiation along the orbit is strongly affected by the variable accretion rate, the magnetic field strength, and by the ambient photon field via dominant IC losses and photon-photon absorption at periastron.
\end{abstract}

Key words. X-rays: binaries - stars: winds, outflows - stars: individual: LS I +61 303 - radiation mechanisms: non-thermal

\section{Introduction}

LS I +61 303 is one of the most intriguing microquasars, which are X-ray binary systems presenting relativistic radio jets. Its discovery as a strongly variable radio source (GT 0236+610) came along with its tentative association with the HE gammaray source 2CG 135+01 (Gregory \& Taylor 1978) detected by COS-B (Hermsen et al. 1977). Moreover, LS I +61 303 was also found to display periodic variability in the radio, infrared, optical, and X-ray bands (Taylor \& Gregory 1982; Martí \& Paredes 1995, MP95 hereafter; Mendelson \& Mazeh 1989; Paredes et al. 1997; respectively). Somewhat unexpectedly, while periastron passage occurs at phase 0.23 (Casares et al. 2005), the X-ray peak occurs at phase $\sim 0.5$ (see, nevertheless, Sidoli et al. 2006), and the radio peak takes place between $\sim 0.45-0.9$. Above $100 \mathrm{MeV}$, EGRET detected variable radiation from the source (3EG J0241+6103, Hartman et al. 1999), and multiwavelength observations also showed unmatched radio/gamma-ray light curves (Kniffen et al. 1997; Tavani et al. 1998). Despite the fact that the available EGRET data is scarce, Massi (2004) pointed out the existence of a peak at periastron, and a second peak at phase 0.5 , in the data presented by Tavani et al. (1998). Very recently, MAGIC detected

\footnotetext{
* Member of CONICET.
}

$\mathrm{TeV}$ emission from LS I +61303 , which is variable and likely associated with the orbital motion (Albert et al. 2006). MAGIC detects the source from phase $\sim 0.4$, around the onset of the radio and X-ray outbursts, until phase $\sim 0.7$, and the $\mathrm{TeV}$ peak occurs at phases $0.5-0.6$, prior to the radio one. The observed $\mathrm{TeV}$ emission is not significant during periastron, contrary to expectations when considering the extrapolation of the EGRET data and accretion models (see, e.g., Bosch-Ramon \& Paredes 2004; Romero et al. 2005).

The companion star in LS I +61 303 is a B0 main sequence star (Paredes \& Figueras 1986) with a slow inhomogeneous equatorial wind forming a circumstellar disk (CD) (Waters et al. 1988). The orbital period of the system is $P=26.4960 \mathrm{~d}$ (Gregory 2002), its eccentricity $e=0.72 \pm 0.15$, the component masses are $\sim 12 M_{\odot}$ for the companion star and $\sim 2.5 M_{\odot}$ for the compact object, for a reasonable inclination angle $i=30^{\circ}$ (Casares et al. 2005). The stellar luminosity is $L_{\star}=10^{38} \mathrm{erg} \mathrm{s}^{-1}$ (Hutchings \& Crampton 1981), and the distance $\sim 2 \mathrm{kpc}$ (Frail \& Hjellming 1991). Massi et al. (2001, 2004) detected mildly relativistic radio jets in LS I +61303 with a velocity $V_{\text {jet }} \sim$ $10^{10} \mathrm{~cm} \mathrm{~s}^{-1}$, pointing to its microquasar nature.

We develop a microquasar model in which the compact object moves on the star equatorial plane accreting CD matter, which is partially ejected as a jet. The jet, embedded in 
the $\mathrm{CD}$, interacts with it, triggering electron acceleration via, e.g., shocks. The jet region where relativistic electrons are injected is adopted as two homogeneous spheres close to the compact object (approaching/receding jet) with diameter $s=R_{\text {orb }} / 3$ each, roughly the CD half height (MP95), although the exact geometry and location are not important provided that the region is homogeneous and $s$ is sufficiently smaller than the orbital distance $\left(R_{\text {orb }}\right)$. Accelerated electrons radiate mainly by synchrotron and IC emission, the latter being significantly absorbed for certain orbital phases above several tens of $\mathrm{GeV}$ via photon-photon annihilation in the stellar and the CD photon fields ${ }^{1}$. We consider convection of particles, thus including adiabatic losses, with the same timescales therein. The model incorporates some contributions from other authors: the relatively low radio and X-ray emission around periastron could be associated with strong IC losses during that orbital phase, avoiding significant synchrotron emission (e.g., Taylor et al. 1996); photon-photon absorption and cascading ${ }^{2}$ could prevent a $\mathrm{TeV}$ peak at phases 0.2-0.3 (see Dubus 2006a; and Bednarek 2006, respectively); EGRET peaks could be linked to accretion maxima (Bosch-Ramon \& Paredes 2004).

\section{The circumstellar disk accretion model}

The accretion rate depends on the relative velocity between the compact object and the CD matter, and on the density of the latter. We refer to MP95 for a characterization of the accretion model within the $\mathrm{CD}$. The measured projected rotational velocity leads to an azimuthal velocity $V_{\phi}=1.13 \times 10^{7} / \sin i \mathrm{~cm} \mathrm{~s}^{-1}$ at one stellar radius $\left(R_{\star}\right)$ (Casares et al. 2005), and angular momentum conservation of the $\mathrm{CD}$ is considered. For the radial velocity of the $\mathrm{CD}$ at the star surface, we have adopted $V_{\mathrm{R}}=3 \times 10^{5} \mathrm{~cm} \mathrm{~s}^{-1}$ to produce an accretion peak at phase $\sim 0.5$, which in the context of our model leads to an X-ray peak at the same phase, as observed (Goldoni \& Mereghetti 1995; Taylor et al. 1996). The CD density at the star surface is fixed to $\rho_{\mathrm{CD}}=10^{-11} \mathrm{~g} \mathrm{~cm}^{-3}$. Following Gregory \& Neish (2002), we add a phenomenological strong CD density cutoff beyond $12 R_{\star}$ $\left(\propto \exp \left[-\left(R_{\mathrm{orb}} / 12 R_{\star}\right)^{10}\right]\right)$ to account for CD dilution at large distances from the star. The resulting normalized accretion rate $\left(\dot{M}_{\text {accr }}\right)$ curve is shown in Fig. 2 at the top. Typical accretion luminosities in this system are about $(0.1-1) \times L_{\text {Eddington, although }}$ we recall this is a first order approximation.

\section{Modeling radiative processes in LS I +61 303}

The emitting region is characterized by its magnetic field $B$ that is assumed to be constant in space and time, and by the photon energy density: $U=U_{\mathrm{CD}}+U_{\star}$, roughly known from observations. To reproduce the observed light curves, we adopt $B=1 \mathrm{G}$. This is not far below equipartition and appears to be a reasonable value. The inner $\mathrm{CD}$ regions, close to periastron passage where CD is likely to be optically thick, are assumed to emit as a black body with temperature $T_{\mathrm{CD}}=1.7 \times 10^{4} \mathrm{~K}$ (MP95) and $U_{\mathrm{CD}}=(4 \sigma / c) T_{\mathrm{CD}}^{4} \sim 700 \mathrm{erg} \mathrm{cm}^{-3}$. In the $\mathrm{CD}$ outer

\footnotetext{
${ }^{1}$ Other external fields of IC seed photons (e.g., the accretion disk) are neglected due to the low soft X-ray luminosities observed in LS I +61 303. Synchrotron self-Compton, and relativistic Bremsstrahlung by jet relativistic electrons going through jet matter, are minor electron cooling channels in our scenario and therefore not considered.

${ }^{2}$ Cascading is not computed here though it would likely increase the $\mathrm{GeV}$ fluxes (Bednarek 2006).
}

regions, emission is likely to be optically thin (Waters 1986), and $U_{\mathrm{CD}}=L_{\mathrm{CD}} / 4 \pi R_{\mathrm{orb}}^{2} c$ there, with $L_{\mathrm{CD}} \sim 10^{37} \mathrm{erg} \mathrm{s}^{-1}$ (Casares et al. 2005). The star is also taken to be a black body with $T_{\star}=2.6 \times 10^{4} \mathrm{~K}$ and $L_{\star} \sim 10^{38} \mathrm{erg} \mathrm{s}^{-1}$ (Hutchings \& Crampton $1981)$, and $U_{\star}=L_{\star} / 4 \pi R_{\text {orb }}^{2} c$. The star photon field dominates all along the orbit except around periastron passage, when the $\mathrm{CD}$ photon field is higher.

The spectrum of the continuously injected electrons, with an isotropic velocity distribution, is $\propto E^{-p}$, with $p=2$ fixed comparing with observed spectra, and $E_{\min }<E<E_{\max }$. $E_{\min }$ is hard to determine and we set it to $1 \mathrm{MeV}$, although more constrained models should also consider a higher lowenergy cutoff (e.g., Bosch-Ramon et al. 2006). $E_{\max }$ is obtained equaling radiative losses in the emitting region with the acceleration rate $\eta q B c$, with an absolute limit induced by the region size: $E_{\max } \leq \eta q B s$. The acceleration efficiency $\eta$ is fixed to 0.01 to explain MAGIC data. This value is well below the electrodynamical limit $(q B c)$ and fits well in the context of shock acceleration theory (see, e.g., Protheroe 1999). The power-law function is normalized to the injected relativistic electron kinetic luminosity, proportional to the accretion rate: $L_{\text {inj }}=L_{\text {norm }} \dot{M}_{18}^{\text {accr }}$ $\left(\dot{M}_{18}^{\text {accr }}=\dot{M}^{\text {accr }} / 10^{18} \mathrm{~g} \mathrm{~s}^{-1}\right)$. To roughly reproduce the observed fluxes at different energies, $L_{\text {norm }}=1.3 \times 10^{35} \mathrm{erg} \mathrm{s}^{-1}$ (considering the two spheres), yielding a $L_{\text {inj }}$ well below $(\sim 1 / 1000)$ the computed available luminosity from accretion.

For the adopted values of $V_{\text {jet }}, B$, and $U$, the electron energy distribution within the considered jet region is led to its steady regime energy on timescales $\ll P$. The timescales associated with radiation processes are shorter than the convection timescale $\left(\tau \sim s / V_{\text {jet }} \sim 10^{2}-10^{3} \mathrm{~s}\right)$ except for the low energy part of the relativistic particle spectrum. Thus, the computed emission in the context of an homogeneous (one-zone) model for a region of size $s$ only provides indicative radio and (IC) X-ray fluxes. Within the considered jet region, the low energy electron spectrum evolution is driven by convection, and the high energy part by radiative losses. A proper determination of both the synchrotron radio and the IC X-ray spectra would require an inhomogeneous jet model. In fact, synchrotron self-absorption has not been computed within the considered jet region to account roughly for the radio emission produced outside it. We compute the synchrotron (Pacholczyk 1970) as well as the inverse Compton emission, and the latter is calculated by taking into account KN and angular effects (Bogovalov \& Aharonian 2000; Dermer \& Böttcher 2006) in the interaction between electrons and photons. The photon-photon opacities due to the ambient photon field are also computed, taking into account angular effects for stellar photons (Gould \& Schréder 1967) and adopting the point-like approximation for the stellar radiation (CD photon field is isotropic when dominant). To account for the angular effects in IC and photon-photon absorption, we characterize the system geometry following Casares et al. (2005). We neglect Doppler effects for the adopted mildly relativistic jet velocity (note that jet orientation is not properly known).

We show the fixed physical quantities of the model in Table 1 and the free parameter values, which have been chosen to roughly reproduce the broadband SED at two significant orbital phases, periastron and phase $\sim 0.5$, as well as the main features of the observed light curves from radio to $\mathrm{TeV}$ (see for comparison Fig. 3 in Chernyakova et al. 2006), in Table 2. The computed SEDs and light curves are presented in Figs. 1 and 2, respectively.

It is worth showing the lepton evolution in the emitting region under the effects of the different loss mechanisms. 
Table 1. Fixed physical quantities in our model.

\begin{tabular}{|c|c|}
\hline Parameter and unit & Value \\
\hline$a$ : orbital semi-major axis $[\mathrm{cm}]$ & $5 \times 10^{12 a}$ \\
\hline$P$ : orbital period $[\mathrm{d}]$ & $26.4960^{b}$ \\
\hline$e$ : eccentricity & $0.72^{c}$ \\
\hline$i$ : inclination angle $\left[{ }^{\circ}\right]$ & $30^{c}$ \\
\hline$M_{\mathrm{x}}$ : compact object mass $\left[M_{\odot}\right]$ & $2.5^{c}$ \\
\hline$M_{\star}:$ stellar mass $\left[M_{\odot}\right]$ & $12^{c}$ \\
\hline$L_{\star}:$ stellar bolometric luminosity [erg s$\left.{ }^{-1}\right]$ & $10^{38 d}$ \\
\hline$T_{\star}:$ stellar surface temperature $[\mathrm{K}]$ & $2.6 \times 10^{4 d}$ \\
\hline$R_{\star}:$ stellar radius $\left[R_{\odot}\right]$ & $10^{e}$ \\
\hline$L_{\mathrm{CD}}: \mathrm{CD}$ bolometric luminosity [erg s ${ }^{-1}$ ] & $10^{37 d}$ \\
\hline$T_{\mathrm{CD}}:$ CD surface temperature $[\mathrm{K}]$ & $1.7 \times 10^{4} e$ \\
\hline$\dot{m}_{\mathrm{w}}:$ stellar mass loss rate $\left[M_{\odot} \mathrm{yr}^{-1}\right]$ & $\sim 10^{-7} e$ \\
\hline$V_{\mathrm{R}}:$ initial $\mathrm{CD}$ radial speed $\left[\mathrm{cm} \mathrm{s}^{-1}\right]$ & $3 \times 10^{5 e}$ \\
\hline$V_{\phi 0}:$ initial $\mathrm{CD}$ azimuthal speed $\left[\mathrm{cm} \mathrm{s}^{-1}\right]$ & $1.13 \times 10^{7} / \sin i^{c}$ \\
\hline$\rho_{\mathrm{CD}}:$ initial CD density $\left[\mathrm{g} \mathrm{cm}^{-3}\right]$ & $10^{-11 e}$ \\
\hline$V_{\text {jet }}:$ convection speed $\left[\mathrm{cm} \mathrm{s}^{-1}\right]$ & $10^{10 f}$ \\
\hline$E_{\min }:$ electron minimum energy $[\mathrm{MeV}]$ & 1 \\
\hline
\end{tabular}

${ }^{a}$ Taylor et al. (1992), ${ }^{b}$ Gregory (2002), ${ }^{c}$ Casares et al. (2005),

${ }^{d}$ Hutchings \& Crampton (1981), ${ }^{e}$ MP95, ${ }^{f}$ Massi et al. (2001, 2004).

Table 2. Adopted values for the free parameters.

\begin{tabular}{ll}
\hline \hline Parameter and unit & Value \\
\hline$B:$ magnetic field [G] & 1 \\
$L_{\text {norm: injection luminosity normalization }\left[\mathrm{erg} \mathrm{s}^{-1}\right]}$ & $1.3 \times 10^{35}$ \\
$\eta:$ acceleration efficiency parameter & 0.01 \\
$p$ : electron energy distribution power-law index & 2 \\
\hline
\end{tabular}

In Fig. 3, the leptonic energy distribution times energy square (electron SED) in the emitting region at different orbital phases is presented. The energies at which the electron energy distribution starts to be dominated by convective losses are indicated. Particles below those energies may radiate their energy further out in the jet, forming for instance the observed radio jets and increasing, to some extent, the radio and X-ray fluxes via synchrotron and IC emission, respectively.

\section{Discussion}

As seen in Fig. 1, the computed SED looks similar to that shown by observations, suggesting that leptonic jet emission can be behind the non-thermal radiation from radio to $\mathrm{TeV}$ energies in LS I+61 303. In the computed SEDs, the effects of the competition between synchrotron and IC losses are seen (see, e.g., at $\mathrm{X}$-rays). The KN IC dominance, especially important during the periastron passage, hardens the synchrotron spectrum beyond UV energies and, less significantly, the IC GeV-TeV spectrum (see also Khangulyan \& Aharonian 2005; for similar treatments on radiative/convective processes, see Khangulyan et al. 2006). We note the dominant effect of the $\mathrm{CD}$ photons during periastron on the over all gamma-ray spectrum.

It is seen in Fig. 2 that the computed radio flux is not particularly high during periastron, although it strongly increases later on, as it is observed (see Ray et al. 1997 for example). The computed X-ray luminosity is the highest around phase 0.5 , like for $\mathrm{TeV}$ radiation, and is similar to what is observed in both energy bands (Harrison et al. 2000 and Albert et al. 2006, respectively). There is a clear peak for computed $\mathrm{GeV}$ emission at periastron passage, as observed, and at phase 0.5 our model predicts just a smooth bump, with flux levels between those found in

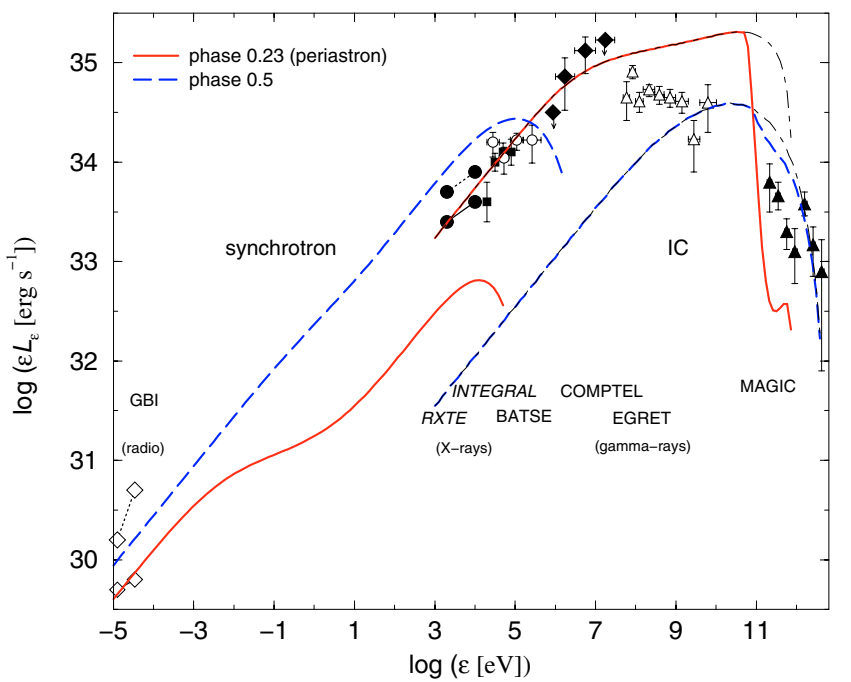

Fig. 1. The computed synchrotron and IC SEDs for jet emission in LS I +61 303 at phases 0.23 (periastron) and 0.5 from radio to TeV energies. The thick lines are SEDs of radiation after attenuation by photon-photon absorption, and the thin lines (above $\sim 100 \mathrm{GeV}$ ) correspond to the production emission. The data points are adapted from Ray et al. (1997, GBI: white diamonds), Harrison et al. (2000, RXTE: black circles), Chernyakova et al. (2006, INTEGRAL: black squares), Harmon et al. (2004, BATSE: white circles), Schönfelder et al. (2000, COMPTEL: black diamonds), Tavani et al. (1998, EGRET: white triangles), and Albert et al. (2006, MAGIC: black triangles). Radio and $\mathrm{X}$-ray data at periastron and phase 0.5 are available from the literature and shown. The COMPTEL and EGRET data correspond to averaged values for different epochs, and the COMPTEL data is possibly affected by other unnoticed gamma-ray sources within the large COMPTEL detection error box. The observed MAGIC spectrum, being the average one, is dominated by emission at phase $\sim 0.5-0.6$ and moderately agrees with the computed SED for the same phase. It is worth noting the different SEDs between periastron and phase 0.5 , which is due to the different electron SEDs (see Fig. 3) and the strong photon-photon opacities.

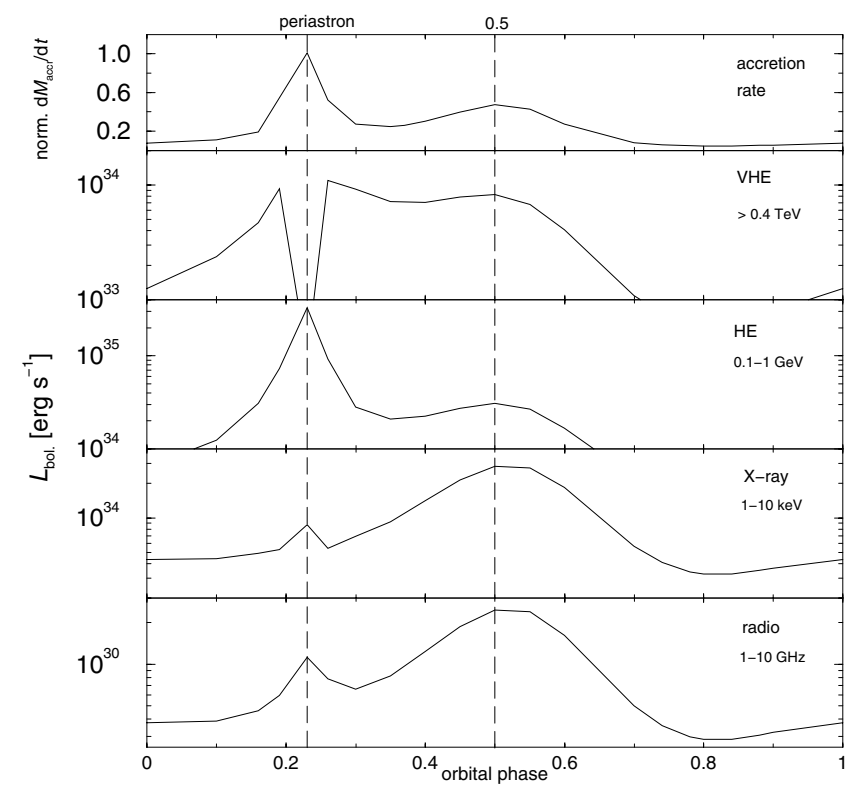

Fig. 2. Top: normalized accretion rate curve. Below: computed light curves of emission in the radio, X-ray, HE and VHE gamma-ray bands.

the different EGRET data sets (Tavani et al. 1998; Massi 2004). The success of assuming electron injection proportional to the 


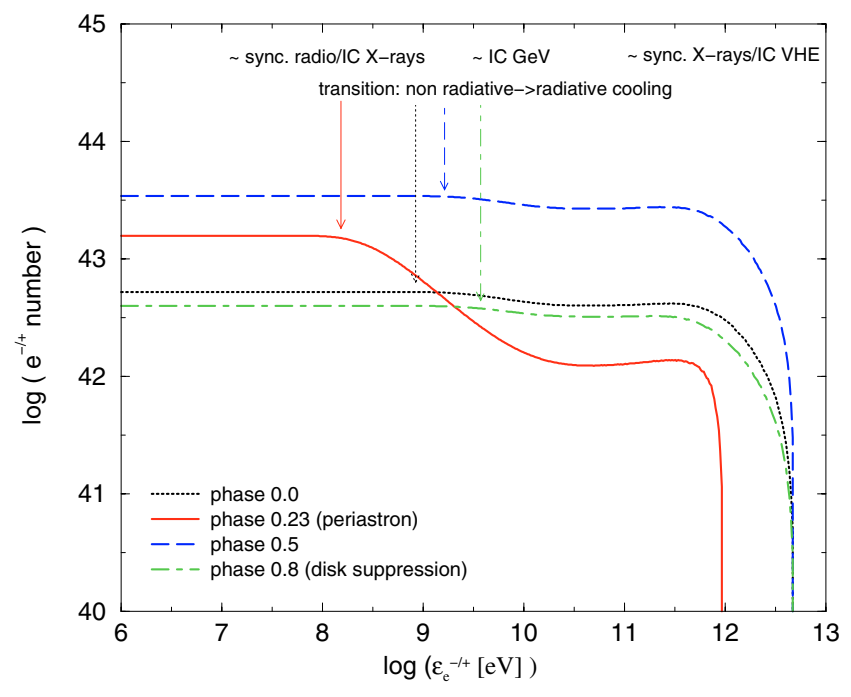

Fig. 3. The electron SED along the orbit (phases 0: dotted line; 0.23: solid line; 0.5: dashed line; and 0.8: large and small dashed line). Note that for different orbital phases the convective, synchrotron, IC Thomson, and IC KN losses dominate in different energy ranges. The transitions from the non-radiative to the radiative regime are shown with arrows, and the typical radiation energy band is shown for the different electron energies. The normalization of the electron SED also changes along the orbit, depending on the injection and cooling efficiency. Moreover, maximum particle energy changes as well due to losses/acceleration balance.

accretion rate curve within the $\mathrm{CD}$ shows the accretion-ejectionemission link (for an alternative scenario, see Dubus 2006b and references therein), and hints at the compact object moving close to the stellar equatorial plane, under a strong CD photon field during periastron passage. The required acceleration efficiency and the injection electron spectrum appear reasonable and seem to point to efficient shock acceleration in the jet. A constant magnetic field along the orbit is the reason why the rapid increase of IC losses at periastron prevents radio and X-ray synchrotron emission from growing in accordance with the accretion rate (see Fig. 2), in agreement with observations.

There are some issues that have not been considered. Since our model focuses on the jet base, the extended radio structure has not been modeled. Also, the treatment of the changes in the radio outburst phase, which could be associated with stellar equatorial flow inhomogeneities (Gregory et al. 1999), is outside the scope of this work. Doppler boosting of the emission, although not very significant for mildly relativistic jets, could be important for temporal evolution if precession occurs (Massi et al. 2004). Actually, the mentioned inhomogeneities could also produce apparent precession via jet deflection along the orbit. In any case, our results are a step forwards to strictly constraining important properties of the source like the electron injection, the dominant cooling channel, the hadronic or leptonic $\mathrm{TeV}$ radiation nature, the system geometry, and how CD affects radiative processes and the jet itself. To accomplish this, future observations with MAGIC and GLAST will be of primary importance.

Acknowledgements. We thank Núria Sidro for helping us when preparing Fig. 1. We thank also Masha Chernyakova and Andrei Neronov for fruitful discussion. V.B-R., J.M.P., and M.R. acknowledge partial support by DGI of the Ministerio de Educación y Ciencia (Spain) under grant AYA-2004-07171C02-01, as well as additional support from the European Regional Development Fund (ERDF/FEDER). During this work, V.B-R has been supported by the DGI of the Ministerio de Educación y Ciencia (Spain) under the fellowship BES2002-2699, M.R. has been supported by CNES and is being supported by a Juan de la Cierva fellowship from Ministerio de Educación y Ciencia. G.E.R is supported by the Argentine Agencies CONICET (PIP 5375) and ANPCyT (PICT 03-13291).

\section{References}

Albert, J., Aliu, E., \& Anderhub, H., et al. 2006, Science, 312, 1771 Bednarek, W. 2006, MNRAS, 368, 579

Bogovalov, S. V., \& Aharonian, F. A. 2000, MNRAS, 313, 504

Bosch-Ramon, V., \& Paredes, J. M. 2004, A\&A, 425, 1069

Bosch-Ramon, V., Romero, G. E., \& Paredes, J. M. 2006, A\&A, 447, 263

Casares, J., Ribas, I., Paredes, J. M., Martí, J., \& Allende Prieto, C. 2005, MNRAS, 360, 1105

Chernyakova, M., Neronov, A., \& Walter, R. 2006, MNRAS, in press [arXiv:astro-ph/0606070]

Dermer, C. D., \& Böttcher, M. 2006, ApJ, 643, 1081

Dubus, G. 2006a, A\&A, 451, 9

Dubus, G. 2006b, A\&A, 456, 801

Frail, D. A., \& Hjellming, R. M. 1991, AJ, 101, 2126

Goldoni, P., \& Mereghetti, S. 1995, A\&A, 299, 751

Gould, R. J., \& Schréder, G. P. 1967, PhRv, 155, 1404

Gregory, P. C. 2002, ApJ, 575, 427

Gregory, P. C., \& Taylor, A. R. 1978, Nature, 272, 704

Gregory, P. C., \& Neish, C. 2002, ApJ, 580, 1133

Gregory, P. C., Peracaula, M., \& Taylor, A. R. 1999, ApJ, 520, 376

Harmon, B. A., Wilson, C. A., \& Fishman, G. J., et al. 2004, ApJS, 154, 585

Harrison, F. A., Ray, P. S., Leahy, D. A., Waltman, E. B., \& Pooley, G. G. 2000, ApJ, 528, 454

Hartman, R. C., Bertsch, D. L., \& Bloom, S. D., et al. 1999, ApJS, 123, 79

Hermsen, W., Swanenburg, B. N., \& Bignami, G. F., et al. 1977, Nature, 269, 494

Hutchings, J. B., \& Crampton, D. 1981, PASP, 93, 486

Khangulyan, D., \& Aharonian, F. A. 2005, in High Energy Gamma-Ray Astronomy: 2nd International Symposium, ed. F. A. Aharonian, H. J. Völk, \& D. Horns (New York: AIP), AIP Conf. Ser., 745, 359

Khangulyan, D., Hnatic, S., Aharonian, F., \& Bogovalov, S. 2006, MNRAS, submitted [arXiv:astro-ph/0605663]

Kniffen, D. A., Alberts, W. C. K., Bertsch, D. L., et al. 1997, ApJ, 486, 126

Martí, J., \& Paredes, J. M. 1995, A\&A, 298, 151 (MP95)

Massi, M. 2004, A\&A, 422, 267

Massi, M., Ribó, M., Paredes, J. M., Peracaula, M., \& Estalella, R. 2001, A\&A, 376,217

Massi, M., Ribó, M., Paredes, J. M., et al. 2004, A\&A, 414, L1

Mendelson, H., \& Mazeh, T. 1989, MNRAS, 239, 733

Pacholczyk, A. G. 1970, Radio Astrophys. (San Francisco: Freeman)

Paredes, J. M., \& Figueras, F. 1986, A\&A, 154, L30

Paredes, J. M., Martí, J., Peracaula, M., \& Ribó, M. 1997, A\&A, 320, L25

Protheroe, R. J. 1999, in Topics in Cosmic-Ray Astrophysics; Horizons in World Physics, ed. M. A. DuVernois (New York: Nova Science Publishers), 230, 247

Ray, P. S., Foster, R. S., Waltman, E. B., Tavani, M., \& Ghigo, F. D. 1997, ApJ, 491,381

Romero, G. E., Christiansen, H., \& Orellana, M. 2005, ApJ, 632, 1093

Schönfelder, V., Bennett, K., \& Blom, J. J., et al. 2000, A\&AS, 143, 145

Sidoli, L., Pellizzoni, A., \& Vercellone, S., et al. 2006, A\&A, in press [arXiv:astro-ph/0606722]

Tavani, M., Kniffen, D., Mattox, J. R., Paredes, J. M., \& Foster, R. 1998, ApJ, 497, L89

Taylor, A. R., \& Gregory, P. C. 1982, ApJ, 255, 210

Taylor, A. R., Kenny, H. T., Spencer, R. E., \& Tzioumis, A. 1992, ApJ, 395, 268

Taylor, A. R., Young, G., Peracaula, M., Kenny, H. T., \& Gregory, P. C. 1996, A\&A, 305, 817

Waters, L. B. F. M. 1986, A\&A, 162, 121

Waters, L. B. F. M., van den Heuvel, E. P. J., Taylor, A. R., Habets, G. M. H. J., \& Persi, P. 1988, A\&A, 198, 200 